\title{
On Development Strategies of TCM Health Tourism
}

\author{
YUAN Zhen, ZHU Zhiying, YANG Hui, YU Ling, CHEN Guang(corresponding author) \\ School of Economics and Management, Jiangxi University of TCM \\ Cguang@foxmail.com
}

\begin{abstract}
Traditional Chinese medicine (TCM) health tourism industry is an emerging industry among China's health service industries. Based on the analysis of the TCM health tourism development status, this paper mainly elaborates the existing problems in the development of TCM health tourism and puts forward relevant development countermeasures, methods and approaches to solve the problems of TCM health tourism development.
\end{abstract}

Keywords-TCM Health Tourism; Development Status; Development Strategies

\section{INTRODUCTION}

One of the driving forces for the development of the health service industry is people's desire to reduce medical costs, while another one is the growing demand of customers for things beneficial to their own health. The typical medical and healthcare tourism refers to a new industry wherein people seek more suitable healthcare services abroad due to the too expensive or imperfect medical services in their places of residence, and this is developed through a combination with leisure tourism. According to the definition of the World Tourism Organization, medical and healthcare tourism is a tourism service with the theme of medical care, disease and health, rehabilitation and cultivation. The medical and healthcare tourism includes two aspects: one is healthcareoriented aspect which achieves the effect of fitness through tourism, including tourism with mountain climbing, drifting, skiing, camping, sunbathing, air bath, forest bath, hot spring bath and other fitness activities; the other is medical-oriented aspect, wherein the tourism industry and medical institutions work together to conduct scientific analysis of the physical condition and state of tourists and to make scientific tourism treatment arrangements. Led by tour guides, medical staff and service staff, the tourists not only make a sightseeing tour, but also learn some healthcare knowledge and adopt some healthcare methods (including the use of TCM healthcare theory and medicated diet) for scientific treatment. At present, the medical and healthcare tourism has become a large-scale industry abroad. The medical and healthcare tourism not only brings health and happiness to those who need the medical and healthcare tourism, but also presents good economic benefits to countries and regions that provide medical and healthcare tourism services [1].

*This paper is research results of college students' innovation and entrepreneurship program. (201610412015)

\section{CONNOTATION OF TCM HEALTH TOURISM}

The TCM health tourism is a new, modern, developing and comprehensive form of health tourism based on the rich TCM resources and culture heritage, as well as a new model of health tourism development based on Chinese medicine culture, TCM industry, tourism industry and health service industry. It is also a new health tourism industry with themes of cultural communication of TCM, healthcare and healthcultivation as well as leisure and tourism, etc [2]. It combines the traditional health-cultivation with tourism and leisure to meet people's growing health tourism needs in a better way. The TCM health theme has the characteristics of regionalism, medical and healthcare nature with strong cultural heritage. The construction of the national demonstration zones of TCM health tourism has a significant impact on promoting the indepth integration of TCM health services and tourism, bringing into full play the TCM's health-cultivation advantages with the theory of harmony between man and nature, forming a large-scale health industry, and ensuring the health needs of Chinese. Since then, Chinese medicine healthy travel has entered Chinese people's lives. In people's health concepts, the medical care and healthcare have been regarded as a regular management item as well as an active topic of concern in their daily life. The passive seeking of medical treatment for illness is no longer the case. Concerns for health, disease prevention and in-advance costs for high-quality life have become a commonplace and consensus in today's social life. The existence of these comprehensive conditions and factors will surely promote China's development of TCM health tourism, and will be a solid link between the tourism industry and the medical and healthcare industry as well as a prominent growth point of them.

As an emerging industry in China's health service industry, TCM health tourism industry is a product combining China's abundant TCM resources and profound TCM culture with the tourism industry. Its rise and development is the results promoted by subconscious based on people's awareness and enhanced understandings of the health concepts and the pursuit of health-cultivation. It is developed with the social and economic development. In people's health concepts, the medical care and healthcare have been regarded as a regular management item as well as an active topic of concern in their daily life. The passive seeking of medical treatment for illness is no longer the case. Concerns for health, disease prevention and in-advance costs for high-quality life have become a commonplace and consensus in today's social life. The existence of these comprehensive conditions and factors will 
surely promote China's development of TCM health tourism, and will be a solid link between the tourism industry and the medical and healthcare industry as well as a prominent growth point of them.

\section{Status Of TCM HeAlTh TOURISM}

\section{A. Good development opportunities faced by TCM health tourism industry}

As an important means to meet people's health-cultivation demands, the TCM health tourism has good development prospects with the supports of various factors. First, the unique TCM resources, the abundant cultural heritage of TCM and TCM technologies provide resources and technical support for the development of TCM health tourism. Second, the government has deepened the structural reforms in various industries and issued a series of policies, guidelines and programs which create a favourable environment for TCM and show state supports. For example, Guidelines on Promoting the Development of TCM Health Tourism, Several Opinions of the State Council on Promoting the Development of Health Service Industry and Plan for the Development of TCM Health Services (2015-2020), etc. propose to exploit the advantages of TCM and to form a group of TCM health tourism service products, put forward the top-level design and strategic goals of the industrial development, and lay a foundation for the specific overall planning. Finally, the upsurge of health-cultivation has provided broad market prospects for development.

\section{B. Cross combination of TCM industry and health tourism industry}

Influenced by the cross combination and upgrading mode among industries, some new operational types for the cross combination of TCM health tourism industry are emerging. For example, the combination of TCM technology and modern rehabilitation tourism gives birth of the "medical tourism with TCM characteristics"; setting scenic spots and spots for healthcare experience in the heritage of TCM gives birth of trips of TCM legacy experience; the combination of TCM education and tourism as well as TCM culture learning during leisure and entertainment gives birth to "popular science education through trip of TCM science popularization education ". These cross combination models have the characteristics of market segmentation and personalized service with a broad range, bring new health tourism experiences and enrich the TCM health tourism industry chain [3].

\section{TCM tourism getting closer to ordinary people's life}

There are many types of popular tourism products with the theme of TCM health in the market today. Parks with a theme of TCM health and the planning and development of TCM tourism parks are combined with ordinary people's daily leisure activities, health-cultivation activities and tourism, which forms some popular health tourism products. In the relationship of people's demands for health and the TCM health tourism industry, the former promotes the latter's continuous innovation and development, while the latter meets the growing needs of the former.

\section{Existing Problems of Traditional Chinese HEALTH TOURISM}

\section{A. Lack of innovation capacity of TCM tourism industry}

China's TCM health tourism industry has the following drawbacks: low additional value, short industrial chain, inadequate exploitation in the depth and breadth of market development, and low resource utilization rates. The reason includes weak innovation capacity and inadequate industryuniversity-research cooperation such as the cooperation in the exploitation of relevant TCM technology and theory system of cultural tourism development. The system combining the features of TCM with the characteristics of health tourism is not formed yet. There are few professionals in the TCM health tourism industry, and they are in a disadvantaged position of market competition. These hinders the development of China's TCM health tourism industry to a certain extent. There are also some urgent problems to be solved in the TCM health tourism. For example, the combination of health-cultivation and tourism has not yet been completed, the scale of development is in its initial stage, the development is not enough, and the brand industry with its own characteristics has not yet been formed. Compared with countries with developed medical tourism, such as Singapore and India, there is still a long way to go in equipment building, service capacity and bonding with leisure activities. Moreover, the complete tourism concepts has not yet formed, developers have not seriously taken the TCM health tourism as a tertiary industry in the Chinese medicine industry: specific development strategies and systematic planning are absent, and development planning and industry standards for the TCM health tourism are not enough; there are few interdisciplinary talents for TCM health tourism, and professional guidance as well as big projects are absent.

\section{B. Not-yet-formed brand}

During the development of TCM health tourism, featured products are the key factor to attract medical tourists. The current TCM health tourism products are mainly in the superficial form of observation and treatment. The services are monotonous, similar and unattractive, and the personalized needs of consumers have not been met yet.

\section{Unmet personalized demands}

The industry related to CTM health tourism is still not perfect. The industrial chain, relevant laws, regulations and industrial standards are defective, such that personalized demands of tourists can not be met. The health tourism market is relatively chaotic, and some enterprises provides illegal and false TCM health tourism services, which hinders the development of the industry. Professionals required for TCM health tourism were in short supply and it is hard to reach a higher service level, resulting in a lag of service concepts and an uneven industrial development. 


\section{DEVELOPMENT STRATEGIES AND SUGGESTIONS ON TCM HEALTH TOURISM}

\section{A. Building a new TCM health tourism pattern}

We shall strives to solve the current deep-seated and superficial problems and create a new pattern of TCM health tourism. A new industry of TCM health tourism with Chinese characteristics shall be fostered. Products featuring characteristic specialty, TCM healthcare, a combination of medical treatment and health-cultivation shall be developed; a health tourism industrial chain shall be built; services with TCM characteristics shall be developed, and tourism resources and TCM resources shall be combined effectively, so as to form a product system with characteristics of TCM health tourism; and the development of routes with a theme of TCM health tourism and featured products mainly to provide TCM medical services shall be encouraged [4].

\section{B. Innovation driving and brand building}

The management innovation shall be strengthened and the capability of technology innovation of TCM health industry shall be improved. Starting from the structural reform of the supply side of TCM health tourism industry, the development of the health tourism industry chain shall be promoted in various links such as resources development, tourism culture and health-cultivation. We shall support social forces in developing the TCM health tourism industry, stimulate the passion for innovation and entrepreneurship of the whole society and speed up the formation of a TCM health tourism development model with innovation as the main driving force. A number of internationally renowned and marketable TCM health tourism enterprises and well-known brands shall be fostered [5].

\section{Developing marketing models with characteristics of TCM health tourism}

Marketing of TCM health tourism should gradually become diversified, innovative, deepened and experiencing. The public awareness of TCM health tourism shall be enhanced with TCM popularization week, TCM health tourism themed festival and TCM popularization tour, etc.; the experience mode of "Internet+" TCM health tourism shall be promoted with the development of "Internet+"; promotional activities such as TCM culture and tourism festival, TCM health-cultivation festival and TCM health tourism forum shall be set and base for experience shall be arranged to promote the development of on-line and off-line experience.

\section{Creating health management model with TCM characteristics}

The entire health management process shall be developed in a scientific way. After collecting health information, conducting physical examination, and evaluating health condition and health risk factors, personalized tourism area, types, projects, methods, etc. shall be planed according to health management theories with TCM characteristics and the features of TCM health tourism projects. Reasonable advices on TCM health tourism projects and activities shall be given. The idea of "preventive treatment of disease" of TCM has been gradually accepted and strengthened by the society. Integrating this idea into the health management meet the essential requirements and development trend of health management. The organic combination of the "preventive treatment of disease" theory of TCM of disease and health management has also been introduced into the development of TCM health tourism. Developers need not have their own medical institutions in the scenic areas or tourist destinations to bring considerate services to tourists; instead, they can help the tourists establish health records, and assist tourists in health management activities such as physical examination, visiting doctors, registration and recovery after illness. Complete health records for tourists can be established based on the network platform and membership system, and specialized personalized health tourism advices and opinions can be provided based of these records, so as to promote the continuous development of TCM health tourism.

\section{CONCLUSION}

TCM diagnosis and treatment methods can be introduced to the modern health management. The basic information of human body shall be collected and sorted; then the basic theory and diagnostic methods of TCM shall be introduced; with four diagnostic criteria of inspection, inquiry, listening and smelling, comprehensive and holistic inspection and evaluation on the physiological conditions of human body can be made. A TCM health management model shall be established. The entire health management process shall be developed scientifically. After collecting health information, conducting physical examination, and evaluating health condition and health risk factors, the four diagnostic criteria shall be used to identify physical fitness and to optimize risk assessment system as well as TCM health intervention measures; guidance for different types of physique shall be provided by professionals such as health managers. Personalized tourism area, types, projects, methods, etc. shall be planed according to health management theories with TCM characteristics and the features of TCM health tourism projects, and reasonable advices on TCM health tourism projects and activities shall be given.

\section{REFERENCES}

[1] LI Donghui, HOU Shengtian. Status and development prospects of healthcare tourism market. Hospital Directors' Forum, 2006

[2] DUAN Zhixiang, LI Zegeng. On strategies of TCM health tourism [J]. Journal of TCM, 2017

[3] ZHANG Miaoying. Limitless potential of combination of TCM health service and tourism China Tourism News, Edition 003, 2016-03-02

[4] Five departments striving to promote health tourism, Chinese Hospital Directors, 2017

[5] REN Hu, CAO Junjin. TCM internationalization under the belt and road initiatives, Bulletin of Science and Technology, 2016. 